\title{
Transculturating for Wordliness
}

English version

\section{Stephanos Stephanides}

\section{(2) OpenEdition \\ Journals}

Electronic version

URL: http://journals.openedition.org/transtexts/161

DOI: 10.4000/transtexts. 161

ISSN: 2105-2549

Publisher

Gregory B. Lee

\section{Printed version}

Date of publication: 1 May 2006

Number of pages: 18-19

ISSN: 1771-2084

\section{Electronic reference}

Stephanos Stephanides, «Transculturating for Wordliness », Transtext(e)s Transcultures 跨文本跨文化 [Online], 1 | 2006, Online since 13 September 2009, connection on 21 April 2019. URL : http:// journals.openedition.org/transtexts/161; DOI : 10.4000/transtexts.161 


\title{
Transculturating for Wordliness by Stephanos Stephanides
}

\begin{abstract}
When I was a student in Britain many years ago, as a migrant out of language and out of place, a "Hellene" and a "post-colonial Cypriot", I was troubled by the linear periodization of my literary studies, which led me from Beowulf to T.S. Eliot and left me asking myself where I belong - looking for the chance connections and turbulence that brought me into that classroom. In the too easy alignment of language, culture, and nation, I was always wanting to move sideward and across, above and below. While my comparatist impulse took me to Hispanic and Luso-Brazilian studies, I found the same linear periodization of Spain and Portugal and peripheralization of the extra-European space as I did in the study of English literature. The subsequent decades led to transformation in academic literary studies with a new emphasis on such issues as the postcolonial and the transcultural. If I have found a space in academia, it is because of the delinquent theorists and writers who brought about this turn to horizontality opening up new possibilities for the diasporic, migrant and hybrid subject. Nonetheless, new hegemonies and orthodoxies arise as we move to a new canon and a new world order that brings with it new forces of singularity, homogenization, technological rationality in an increasingly global experience, in supranational states, and this brings new challenges for the transcultural.

The idea of transculturation designates a space where diverse forces and influences cross that may derive from the nation state, nature, or the market. As a disorderly flow of influences caught between state regulation and cultural desire, transculturation in itself is neither to be celebrated nor condemned. It may impose closure and fix identity, and declare the nation state to be adequate to that identity or alternatively remain open, identity unfixed, establishing a flow between diverse groups neither coerced nor constrained by the nation state. While transculturation seems to have gained momentum in the era of postmodernity and in an age of globalization, it is worth recalling that the term was coined by Fernando Ortiz, the Cuban writer and anthropologist, in the 1940s. 1

As the projects of modernity and colonialism have been closely connected, Ortiz's notion comes as a post-colonial negotiation of Cuban culture autonomy in modernity. It is noteworthy that a mode of aesthetic expression we associate nowadays with postmodernism was articulated by writers within the context of colonial cosmopolitanism - not only in Latin America but elsewhere - and seems to anticipate a postmodern moment of the periphery when the intelligentsias outside the West mastered modernity to turn it into something of their own. Peripheral modernities diverge from European prototypes according to the spatio-temporal specificities of individual societies. One may therefore speak of multiple modernities both within and outside Europe. Usually the idea of the centre refers to the geopolitical status of
\end{abstract}

1 Fernando Ortiz, [Trans. Harriet de Onís], Cuban Counterpoint: Tobacco and Sugar, Durham, Duke University Press, 1995. 
the West where modernity began, but the periphery never shared with the Centre the specific pre-modern condition that would shape the way the peripheries developed, and there is not simply a linear movement from modernity to post-modernity. Different areas of the periphery do not share the same trajectory and culture has played a diverse role in cementing the bonds of the nation-state or served as a catalyst for its transformation. Translation and colonialism have played a significant role in the emergence of Indian vernaculars as literary languages such as we see in the example of the so-called Bengali renaissance. In its Caribbean and Latin American context, the transcultural is linked to the processes of creolization in language and culture. The idea that modernity in the periphery can only be a copy subtends Latin American modernismo itself. We find this in the stance of refusal or "anarchistic aesthetic" of a poet such as Ruben Dario, which may be seen as a strategic exodus from the mundane reality that the State conditions, and also in the use of translation as parody and pastiche as constant decentring that we find in Borges, Brazilian antropofagismo, or Cuban criollismo. Transculturation thus describes history as it takes place behind the back of the state. The coining of the term by Ortiz seems to belatedly name a process that had already been part of the Caribbean and Latin American modernist artistic and intellectual imaginary in its effort to reclaim the values that precede the State (colonial or postcolonial) or found anew values outside its field of influence.

Artists and Intellectuals have played key roles at the intersection of culture and state, and the politics in the translation and mediation of cultural demands and desire. However this role is not unambiguous and the transculturation and translation process is in no way simple or transparent. Moreover, there are numerous ways by which artists and the intelligentsia appropriate resources to generate and sustain distance from culture and state as they may veer between a bohemian condition and a Mandarin status. Are they agents or transculturation or do they articulate its effects ? To what extent are they able to disinvest or distance themselves from the State and State culture in a quest for the ideal of pure autonomy ? The State itself through its academic institutions, museums and learned societies confirms the virtue of their artistic and intellectual labour. The neo-liberal State cedes some discursive legitimacy to intellectuals and artists, but rewards the services of technocrats rather than intellectuals who will make a stand or bridge between State and people.

While transculturation may have been a thorn in the side of the nation state and nationalism, in an age of global and trans-national economy and politics, one has to ask which State or state. Recent scholarship often claims the distinction between globality and worldliness (as implied in Heidegger's idea of the worlding), reclaiming for the latter the world making function in human experience and intentionality, historical contingency, and the relentless sensuality of nature. In contrast to the inexorable movement of capital, it is worth returning to the attempts of modernist artists and intellectuals to achieve autonomy through transculturation. If the term is often appropriated by the globalization process, we must reclaim it for the world and the worldly text. 


\section{Verbe, Monde et Transculturation}

Lorsque j'étais étudiant en Grande-Bretagne il y a plusieurs années, un migrant hors de sa langue et de son monde, " un Hellène » et " un Chypriote postcolonial », j'ai été troublé par la périodisation linéaire de mes études littéraires qui allait de Beowulf à TS Eliot et me laissait perplexe quant à ma propre appartenance - à la recherche des connections fortuites et des turbulences par lesquelles j'étais dans cette classe. Dans cet alignement trop simple de langage, culture et nation, j'avais toujours envie d'aller à côté et à travers, par dessus et en dessous. Alors que mes ardeurs de comparatiste m'avaient entraîné vers les études hispaniques et luso-brésiliennes, je retrouvais la même périodisation linéaire de l'Espagne et du Portugal et le même rejet vers la périphérie de l'espace extra-européen que dans les études de littérature anglaise. Pendant les décennies suivantes, les études littéraires académiques connurent des transformations, une importance nouvelle étant accordée à des thématiques telles que celles du postcolonial et du transculturel. Si j'ai trouvé ma place au sein de l'académie, c'est grâce aux écrivains et théoriciens délinquants qui ont amené ce tournant vers l'horizontalité et ouvert de nouvelles possibilités pour les sujets diasporiques, migrants et hybrides. Néanmoins, de nouvelles hégémonies et orthodoxies émergent tandis que nous évoluons vers un nouveau canon et un nouvel ordre du monde qui draine avec lui de nouvelles forces de singularité, d'homogénéisation et de rationalité technologique dans le cadre d'une expérience de plus en plus globale et à l'intérieur d'Etats supranationaux, ce qui entraîne de nouveaux défis pour le transculturel.

L'idée de transculturation désigne un espace où des forces et influences diverses se croisent, qu'elles proviennent de l'Etat-nation, de la nature ou du marché. En tant que flux désordonné d'influences prisent entre les règlements étatiques et le désir culturel, la transculturation, en soi, n'est ni à célébrer ni à condamner. II se peut qu'elle impose l'enfermement et une identité figée, et qu'elle déclare que l'Etat-nation convient à cette identité, ou bien elle peut rester ouverte, sans que l'identité soit figée, établissant ainsi un flux entre des groupes différents, ni contraint ni restreint par l'Etat-nation.

La transculturation semble pourtant avoir gagné du terrain dans l'ère de la postmodernité et à une époque de globalisation, il est nécessaire de rappeler que ce terme a été inventé dans les années 1940 par Fernando Ortiz, écrivain et anthropologue Cubain. 2

Les projets de la modernité et du colonialisme étant intimement liés, la notion introduite par Ortiz intervient en tant que négociation postcoloniale de l'autonomie culturelle cubaine dans la modernité. II convient de noter que l'un des modes d'expression esthétique que nous associons aujourd'hui au postmodernisme a été élaboré par des écrivains immergés dans le contexte du cosmopolitisme colonial - non seulement en Amérique Latine mais aussi

1 Fernando Ortiz, [Trans. Harriet de Onís], Cuban Counterpoint: Tobacco and Sugar, Durham, Duke University Press, 1995. 
ailleurs- et semble anticiper un moment postmoderne de la périphérie où les intelligentsias extérieures à l'Ouest ont négocié la modernité pour se l'approprier. Les modernités périphériques divergent des prototypes européens selon les spécificités spatio-temporelles de chaque société. On peut alors parler de modernités multiples, qu'elles soient intérieures ou extérieures à l'Europe. L'idée de centre correspond généralement au statut géopolitique de l'Ouest en tant que point de départ de la modernité. Cependant la périphérie n'a jamais été dans les conditions pré-modernes spécifiques au centre qui étaient censées déterminer le développement des périphéries, et le mouvement de la modernité vers la postmodernité n'est pas simplement linéaire. Les différents espaces de la périphérie ne suivent pas la même trajectoire et la culture a tantôt participé de diverses manières à la solidification des contours de l'Etat-nation, tantôt accéléré sa transformation. La traduction et le colonialisme ont joué un rôle significatif dans l'émergence de vernaculaires indiens en tant que langages littéraires ainsi que nous le montre l'exemple de la « renaissance du Bengali ». Dans le contexte des Caraïbes et de l'Amérique Latine, le transculturel est lié aux processus de créolisation du langage et de la culture. L'idée que la modernité dans la périphérie ne peut être qu'une reproduction sous-tend le Modernismo d'Amérique Latine. On la retrouve dans la position de refus ou « esthétique anarchique » d'un poète tel que Ruben Dario, qui peut être vue comme une fuite stratégique, un éloignement de la terne réalité déterminée par l'Etat, mais aussi dans l'usage de la traduction comme parodie et pastiche, décentrement permanent que l'on trouve chez Borges, dans l'anthropofagisme brésilien ou le Criollismo Cubain. C'est ainsi que la transculturation décrit l'histoire telle qu'elle se déroule à l'arrière de l'Etat. L'invention de ce terme par Ortiz semble nommer tardivement un processus qui faisait déjà partie de l'imaginaire intellectuel et artistique moderniste des Caraïbes et de l'Amérique Latine dans le sens où cet imaginaire s'efforçait de récupérer des valeurs antérieures à l'Etat (colonial ou postcolonial) et d'établir de nouveau des valeurs en dehors de sa sphère d'influence.

Artistes et Intellectuels ont joué des rôles clés à l'intersection de l'Etat et de la culture, dans les politiques de traduction et de médiation des demandes et désirs culturel. Néanmoins, ce rôle n'est pas dénué d'ambiguité, et le processus de transculturation et de traduction n'est en aucune manière simple ou transparent. En outre, les artistes et l'intelligentsia s'approprient de diverses manières des ressources afin de générer et maintenir une distance par rapport à la culture et à l'Etat comme s'ils étaient écartelés entre une condition de bohémien et un statut de mandarin. Sont-ils des agents de la transculturation ou bien la voix de ces effets? A quel point sont-ils capables de se désengager et de se mettre à distance de l'Etat et de la culture d'Etat dans leur quête de l'idéal d'une autonomie parfaite ? L'Etat, lui-même, à travers ses institutions académiques, ses musées, ses groupes d'érudits, reconnaît la valeur de leur travail artistique et intellectuel. L'Etat néo-liberal concède une relative légitimité discursive aux intellectuels et aux artistes, mais il récompense les services des technocrates plutôt que ceux des intellectuels qui pourraient servir de support ou de pont entre l'Etat et le peuple.

II se peut que la transculturation ait été dérangeante pour l'Etat-nation et pour le nationalisme; demandons-nous cependant, à une époque d'économie et de politique trans-nationales et globales, de quel Etat ou état il s'agit. On trouve fréquemment dans des travaux récents l'affirmation selon laquelle il faut distinguer la mondialité (globality) de la mondanéité(worldliness) (dans le sens de worlding, selon Heidegger), la capacité de l'expérience humaine et de l'intentionnalité à faire le monde, la contingence historique, ainsi que l'incessante sensualité de la nature, étant réservées à la mondanéité. Par opposition au 
mouvement inexorable du capital, il est intéressant de retrouver les tentatives des artistes et intellectuels modernistes de parvenir à l'autonomie par le biais de la transculturation. Bien que ce terme soit souvent appliqué au processus de mondialisation, nous devons le revendiquer pour le monde et pour le texte mondial.

\section{指向于世界性的跨文化}

$\begin{array}{ll}\text { 多年以前在英国, 当我还是个学生的时候, 作为一个远离乡音、背井离乡的的希腊人”、一个“后殖民时代的赛路斯人”, 我被自己文学研究 } & 0 \\ \text { 移民, 一个 }\end{array}$ 中线性的时代划分所困惑着, 它将我从贝奥伍夫（Beowulf）引向艾略特（T.S. Eliot），让我不断地问自己哪里才是我的归属- - 寻找把我带入到那间教室的 机缘与骚动。在语言、文化与民族间极为容易的联姻中, 我总是想前后左右、 上上下下地摇摆。当我比较主义学者式的冲动使我对西班牙与葡萄牙一巴西研 究产生兴趣的时候, 我发现了如同我在英语文学研究中看到的一样, 西班牙和 葡萄牙这两个国家线性的时代划分和欧洲外空间的周边化。随后的几十年中, 学术界的文学研究转向了一种新的侧重点, 如后殖民时代与跨文化这样的问 题。如果说我已经在学术界中找到了立足点的话, 那是因为那些失职的理论家 与作家们, 是他们把这一切转向了为离散、移民与混杂等主题开创新的可能性 的境地。但是, 当我们走向新的规则与新的世界秩序的时候, 新的霸权与所谓 的正统也在崛起, 前者随之在一个不断增长的全球性经验中, 在超民族国家中 带来了某些单一性、霸权化与技术理性方面的新力量, 而这一切为跨文化提出 了新的挑战。

跨文化观念意味着一种空间, 在那里各种力量与影响超越了从民族国家、自然 或市场衍生而来的某些东西。作为一种拘于国家管制与文化意愿之间失范的影 响潮流, 跨文化就其本身而言既不值得庆贺也不应受到谴责。它或许会强制终 止或固定身份特性, 并宣称民族国家适宜于那种身份, 要么或者只能是保持开 放状态，不固定身份，由民族国家在各种既非强迫又非压制性的群体间建立一 种流动状态。尽管跨文化在后现代性时期和全球化时代已获得了某种势头, 但 值得提醒的是这一术语是由古巴作家、人类学家欧提斯（Fernando Ortiz）在二 十世纪四十年代杜撰的。3

由于现代性与殖民主义计划早已紧密地联系在一起, 欧提斯的概念是作为一种 在现代性背景下古巴文化自治的后殖民主义协商出现的。值得注意的是, 今天 我们将之与后现代主义相联系的审美表述的一种模式早已被作家们在殖民地的 世界主义语境中清楚地表达了出来一一不仅在拉丁美洲, 还有其他地方, 并且 在西方世界之外的知识分子界掌握了现代性并要将之转化进他们自己的某些东 西中的时候，这种模式似乎预见到了周边的一个后现代性时刻。依照个体社会 的时空特性而言，周边的诸多现代性与欧洲原形有所不同。因此，我们或许可 以讲在欧洲内外有多种现代性。通常而言, 中心观念是指作为现代性发轫之地 的西方世界的地缘政治地位，但是周边地区从未与“中心”共享过特定的前现代 性条件, 这一条件可能会形成周边诸多地区发展的道路, 而且从现代性到后现 代性的过程中, 并非只是一种线性运动。周边不同的地区并没有共享同样的发 
展转变, 而且文化在巩固民族-国家的联结方面扮演了不同的角色，或者作为 其变革的催化剂。翻译与殖民主义在印度方言变成为文学语言的过程中发挥了 重大的作用，比如我们在所谓的孟加拉复兴（Bengali renaissance）这一实例中所 知道的。在其加勒比海与拉丁美洲语境中，跨文化同语言与文化中的混杂过程 密切相关。认为周边地区的现代性只能是一种复制的观念恰恰与拉丁美洲的现 代主义本身相反。我们可以在如同达利奥（Ruben Dario）这样的诗人的拒绝姿 态或“无政府主义的审美标准”中发现这一点, 这位诗人可以被看作是一个出自 这个国家以之作为条件的世俗现实中的策略性的逃脱者，也可以在翻译作为持 续进行非中心化的拙劣仿制与模仿使用中发现这一点，而后者我们可以在博尔 赫斯（Borges）的作品，及巴西的食人习俗（antropofagismo）或古巴的克里欧由 主义（criollismo）中找到。因此，跨文化将历史描述成其发生在国家的背后。 欧提斯对于这一术语的杜撰看起来追命了早已成为巴西和拉丁美洲现代主义艺 术和知识分子想象的一部分的一种过程，这些想象出现于其重申那些先于国家 （殖民的或后殖民的）的价值观或在其影响范围之外重铸价值观的努力当中。 艺术家与知识分子在文化与国家的交叉过程中扮演了重要的角色，政界则在文 化要求与意愿间的转化与调解方面发挥了重要的作用。然而, 这种角色与作用 并不是明确的，跨文化与翻译过程也绝不是简单或透明的。而且，有很多途径 是艺术家与知识分子界借以调动资源以产生或维持与文化和国家间的距离, 因 为他们可能会在一种放任不羁的状况与一种官僚权位之间有所转向。他们是中 介或跨文化吗? 或者他们能清楚表明其影响吗? 在一种对于纯粹自治之理想的 寻求中，他们能在多大程度上解除自己被国家和国家文化的包围或疏远自身与 其之间的距离? 国家本身可以通过其学术机构与制度、博物馆及学术团体来巩 固他们艺术与智力劳动成果的优秀品质。新的自由国家会让与一部分话语合法 性给知识分子与艺术家, 但却奖赏技术专家而非知识分子的服务, 后者会在国 家与人民之间设置某种阻碍或起到桥梁作用。

在一个全球性与跨国化的经济与政治时代，尽管站在民族国家和民族主义的立 场上, 跨文化可能一直是一种荆棘, 但我们不得不要问是哪个国家，在何种情 况下? 近来很多学术研究经常呼吁要分清全球性与世界性（worldliness）（如同 海德格尔（Heidegger）在有关worlding观念中的意义）之间的区别，将运作于人 性的经验与目的性、历史的偶然性与无情的本性肉欲之中的世界归还给后者。 与不可阻挡的资本运作相对照，有必要再回到现代主义艺术家与知识分子通过 跨文化而要实现自治的努力之中。如果这一术语经常被全球化过程所使用的 话, 那么我们必须将之归还给世界和世界性的文本。 